УДК 621.396 .69

\title{
КОМПЛЕКСНАЯ ЭФФЕКТИВНАЯ ДИЭЛЕКТРИЧЕСКАЯ ПРОНИЦАЕМОСТЬ МИКРОМЕХАНИЧЕСКИ ПЕРЕСТРАИВАЕМЫХ МИКРОПОЛОСКОВЫХ ЛИНИЙ
}

\author{
Е. А. ЦЫБА, И. П. ГОЛУБЕВА, В. А. КАЗМИРЕНКО, Ю. В. ПРОКОПЕНКО
}

\author{
Национальный технический университет Украинь \\ «Киевский политехнический институт им. Игоря Сикорского», \\ Украина, Киев, 03056, пр-т Победы 37
}

\begin{abstract}
Аннотация. Рассмотрено влияние физико-топологических параметров управляемой микрополосковой линии, в которой изменение характеристик достигается путем перемещения сигнального электрода над подложкой, на перестройку эффективной диэлектрической проницаемости и потери электромагнитной энергии в линии, выраженные в форме комплексной диэлектрической проницаемости. Установлены пути повышения чувствительности изменения эффективной диэлектрической проницаемости к перемещениям сигнального электрода и уменьшения потерь. Установлены предельно достижимые характеристики перестройки и потерь. Представленные расчеты коэффициента передачи и эффективной диэлектрической проницаемости согласуются с результатами экспериментов. Результаты могут быть использованы для проектирования управляемых резонансных элементов и фазовращателей с применением электрически управляемых микроперемещателей, таких как пьезо- и электрострикционных актюаторов или микроэлектромеханических систем. Благодаря применению инвариантных отношений физико-топологических параметров приведенные расчеты пригодны для оценки показателей перестройки и потерь устройств с микромеханическим управлением в широком диапазоне рабочих частот и с применением широкого спектра материалов.
\end{abstract}

Ключевые слова: микрополосковая линия; микромеханическая перестройка; эффективная диэлектрическая проницаемость; потери

\section{ВВЕДЕНИЕ}

Несмотря на прогресс в разработке твердотельных компонентов для работы в диапазоне СВЧ, исследование и разработка механически перестраиваемых элементов СВЧ устройств не только остается актуальной задачей [1], но и получает новое качество с внедрением таких передовых технологий, как микроэлектромеханические системы (МЭМС) [2] и 3D-печать [3]. Управляемые микрополосковые линии (МПЛ) применяются для создания перестраиваемых устройств СВЧ, таких как фазовращатели [4] и фильтры $[5,6]$. Эти конструкции позволяют достичь перестройки рабо- чей частоты и фазы в широких пределах при сохранении, а иногда и с улучшением добротности, чего нельзя достичь с использованием твердотельных приборов [7-9].

Принцип действия таких перестраиваемых устройств заключается в изменении электрического пути электромагнитной волны, которое может достигаться как изменением геометрических размеров частей устройств, так и изменением их эффективных параметров. В отличие от традиционного способа настройки и перестройки, последний способ открывает возможности для реализации перестройки 


\section{БИБЛИОГРАФИЧЕСКИЙ СПИСОК}

1. Kurudere, S.; Erturk, V. B. Novel microstrip fed mechanically tunable combline cavity filter. IEEE Microwave and Wireless Components Lett., Vol. 23, No. 11, pp. 578-580, Nov. 2013. DOI: 10.1109/LMWC.2013. 2281432.

2. Fouladi, S.; Huang, F.; Yan, W. D.; Mansour, R. R. High-Q narrowband tunable combline bandpass filters using MEMS capacitor banks and piezomotors. IEEE Trans. Microwave Theory Tech., Vol. 61, No. 1, pp. 393-402, Jan. 2013. DOI: 10.1109/TMTT.2012.2226601.

3. Gillatt, Brendan T. W.; D'Auria, Mario; Otter, William J.; Ridler, N. M.; Lucyszyn, S. 3-D printed variable phase shifter. IEEE Microwave and Wireless Components Lett., Vol. 26, No. 10, pp. 822-824, 2016. DOI: $10.1109 /$ LMWC.2016.2604879.

4. Romano, P.; Araromi, O.; Rosset, S.; Perruisseau-Carrier, J.; Shea, H.; Mosig, J. R.; Ramon, Juan. Low-loss millimeter-wave phase shifters based on mechanical reconfiguration. Proc. of Progress In Electromagnetics Research Symp., PIERS, 6-9 Jul. 2015, Prague. Prague, 2015. URI: https://infoscience.epfl.ch/ record $/ 210386$.

5. Bouyge, D.; Mardivirin, D.; Bonache, J.; Crunteanu, Aurelian; Pothier, Arnaud; Duran-Sindreu, Miguel; Blondy, Pierre; Martin, Ferran. Split ring resonators (SRRs) based on micro-electro-mechanical deflectable cantilever-type rings: application to tunable stopband filters. IEEE Microwave and Wireless Components Lett., Vol. 21, No. 5, pp. 243-245, 2011. DOI: 10.1109/LMWC.2011.2124450.

6. Poplavko, Y.; Prokopenko, Y.; Pashkov, V.; Molchanov, V.; Golubeva, I.; Kazmirenko, V.; Smigin, D. Low loss microwave piezo-tunable devices. Proc. of 36 th European Microwave Conf., 10-15 Sept. 2006, Manchester, UK. IEEE, 2006, pp. 657-660. DOI: 10.1109/EUMC. 2006.281496.

7. Ni, Jia; Hong Jiasheng. Varactor-tuned microstrip bandpass filters with different passband characteristics. IET Microwaves, Antennas \& Propag., Vol. 8, No. 6, pp. 415-422, 2014. DOI: 10.1049/iet-map.2013.0474.

8. Karim, M. F.; Guo, Y.-X.; Chen, Z. N.; Ong, L. C. Miniaturized reconfigurable and switchable filter from UWB to $2.4 \mathrm{GHz}$ WLAN using PIN diodes. IEEE MTT-S Int. Microwave Symp. Dig., 7-12 Jun. 2009, Boston, MA, USA. IEEE, 2009, pp. 509-512. DOI: 10.1109/MWSYM. 2009.5165745.

9. Jin, Zhang; Ortiz, S.; Mortazawi, A. Design and performance of a new digital phase shifter at X-band. IEEE Microwave and Wireless Components Lett., Vol. 14, No. 9, pp. 428-430, Sept. 2004. DOI: 10.1109/LMWC.20 04.832049 .

10. Yun, T.-Y.; Chang, K. Analysis and optimization of a phase shifter controlled by a piezoelectric transducer. IEEE Trans. Microwave Theory Tech., Vol. 50, No. 1, P. 105-111, 2002. DOI: 10.1109/22.981254.

11. Golubeva, I.; Kazmirenko, V.; Sergienko, P.; Prokopenko, Y. Effective permittivity in tunable microstr- 
ip and coplanar lines. Proc. of XXXII Int. Sci. Conf. on Electronics and Nanotechnology, ELNANO, 10-12 Apr 2012, Kyiv, Ukraine. Kyiv, 2012, pp. 69-70. URI: http://www.journals.kpi.ua/publications/text/69 $70 \quad 2012$ .pdf.

12. Прокопенко, Ю.В. Пределы управляемости диэлектрической неоднородности, расположенной между металлическими плоскостями. Технология $u$ конструирование в электронной аппаратуре, № 6, С. 16-20, 2012. URI: http://www.tkea.com.ua/tkea/2012/6 2012/st 04.htm.

13. Gupta, K. C. Microstrip Lines and Slotlines, 2nd ed. Artech House, 1996. 535 p.
14. Сергиенко, П.Ю.; Казмиренко, В.А.; Чернов, А.С.; Прокопенко, Ю.В.Добротность перестраиваемых микрополосковых резонаторов. Известия вузов. Радиоэлектроника, Т. 59, № 2, C. 52-60, 2016. DOI: 10.20535/S0021347016020060.

15. Sergienko, P.; Golubeva, I.; Prokopenko, Y. Loss in tunable microstrip lines. Proc. of IEEE 34th Int. Conf. on Electronics and Nanotechnology, ELNANO, 15-18 Apr. 2014, Kyiv, Ukraine. IEEE, 2014, pp. 97-100. DOI: 10.1109/ELNANO.2014.6873972.

16. Ахиезер, А. И.; Ахиезер, И. А. Электромагнетизм и электромагнитные волны. М.: Высшая школа, 1985, C. 324-325. 